\title{
Numerical Solution of Singularly Perturbed Delay Differential Equations with Layer Behavior
}

\author{
F. Ghomanjani, ${ }^{1}$ A. Kılıçman, ${ }^{2}$ and F. Akhavan Ghassabzade ${ }^{1}$ \\ ${ }^{1}$ Department of Applied Mathematics, Faculty of Mathematical Sciences, Ferdowsi University of Mashhad, Mashhad, Iran \\ ${ }^{2}$ Department of Mathematics and Institute for Mathematical Research, University Putra Malaysia (UPM), \\ 43400 Serdang, Selangor, Malaysia
}

Correspondence should be addressed to A. Kılıçman; akilic@upm.edu.my

Received 4 September 2013; Accepted 24 December 2013; Published 16 January 2014

Academic Editor: Aref Jeribi

Copyright (C) 2014 F. Ghomanjani et al. This is an open access article distributed under the Creative Commons Attribution License, which permits unrestricted use, distribution, and reproduction in any medium, provided the original work is properly cited.

\begin{abstract}
We present a numerical method to solve boundary value problems (BVPs) for singularly perturbed differential-difference equations with negative shift. In recent papers, the term negative shift has been used for delay. The Bezier curves method can solve boundary value problems for singularly perturbed differential-difference equations. The approximation process is done in two steps. First we divide the time interval, into $k$ subintervals; second we approximate the trajectory and control functions in each subinterval by Bezier curves. We have chosen the Bezier curves as piecewise polynomials of degree $n$ and determined Bezier curves on any subinterval by $n+1$ control points. The proposed method is simple and computationally advantageous. Several numerical examples are solved using the presented method; we compared the computed result with exact solution and plotted the graphs of the solution of the problems.
\end{abstract}

\section{Introduction}

In recent years, there has been a growing interest in the singularly perturbed delay differential equation (see [1-4]). A singularly perturbed delay differential equation is an ordinary differential equation in which the highest derivative is multiplied by a small parameter and involving at least one delay term. Such types of differential equations arise frequently in applications, for example, the first exit time problem in modeling of the activation of neuronal variability [5], in a variety of models for physiological processes or diseases [6], to describe the human pupil-light reflex [7], and variational problems in control theory and depolarization in Stein's model [8]. Investigation of boundary value problems for singularly perturbed linear second-order differentialdifference equations was initiated by Lange and Miura [ 5 , 9, 10]; they proposed an asymptotic approach in study of linear second-order differential-difference equations in which the highest order derivative is multiplied by small parameters. Kadalbajoo and Sharma [11-14] discussed the numerical methods for solving such type of boundary value problems. Amiraliyev and Erdogan [15] and Amiraliyeva and Amiraliyev [16] developed robust numerical schemes for dealing with singularly perturbed delay differential equation.

In the present work we suggest a technique similar to the one which was used in $[17,18]$ for solving singularly perturbed differential-difference equation with delay in the following form (see [13]):

$$
\begin{gathered}
\epsilon y^{\prime \prime}(t)+a(t) y^{\prime}(t-\delta)+b(t) y(t)=f(t), \quad 0<t<1, \\
y(t)=\phi(t), \quad-\delta \leq t \leq 0, \\
y(1)=\gamma,
\end{gathered}
$$

where $\epsilon$ is small parameter, $0<\epsilon \ll 1$, and $\delta$ is also a small shifting parameter, $0<\delta \ll 1, a(t), b(t), f(t)$, and $\phi(t)$ are assumed to be smooth, and $\gamma$ is a constant. For $\delta=0$, the problem is a boundary value problem for a singularly perturbed differential equation and then as the singular perturbation parameter tends to zero, the order of the corresponding reduced problem is decreased by one, so there will be one layer. It may be a boundary layer or an 
interior layer depending on the nature of the coefficient of the convection term.

The current paper is organized as follows. In Section 2, function approximation will be introduced. Numerical examples will be stated in Section 3. Finally, Section 4 will give a conclusion briefly.

\section{Function Approximation}

Consider the problem (1). Divide the interval $\left[t_{0}, t_{f}\right]$ into a set of grid points such that

$$
t_{i}=t_{0}+i h, \quad i=0,1, \ldots, k,
$$

where $h=\left(t_{f}-t_{0}\right) / k, t_{f}=1, t_{0}=0$ and $k$ is a positive integer. Let $S_{j}=\left[t_{j-1}, t_{j}\right]$ for $j=1,2, \ldots, k$. Then, for $t \in S_{j}$, the problem (1) can be decomposed to the following suboptimal control problems:

$$
\begin{gathered}
\epsilon y_{j}^{\prime \prime}(t)+a(t) y_{-k_{2}+j}(t-\delta)+b(t) y_{j}(t)=f(t), \quad t \in S_{j}, \\
y_{j}(t)=\phi(t), \quad-\delta \leq t \leq t_{0}, \quad j=1,2, \ldots, k, \\
y_{k}(1)=\gamma,
\end{gathered}
$$

where $y_{j}(t)$.

We mention that $y_{-k_{2}+j}(t-\delta)$ is defined where $(t-\delta) \epsilon$ $\left[t_{-k_{2}+j-1}, t_{-k_{2}+j}\right]$. Also

$$
k_{2}= \begin{cases}\frac{\delta}{h}, & \frac{\delta}{h} \in \mathbb{N}, \\ \left(\left[\frac{\delta}{h}\right]+1\right), & \frac{\delta}{h} \notin \mathbb{N},\end{cases}
$$

where $[\delta / h]$ denotes the integer part of $\delta / h$.

Let $y(t)=\sum_{j=1}^{k} \chi_{j}^{1}(t) y_{j}(t)$ where $\chi_{j}^{1}(t)$ is the characteristic function of $y_{j}(t)$ for $t \in\left[t_{j-1}, t_{j}\right]$. It is trivial that $\left[t_{0}, t_{f}\right]=$ $\bigcup_{j=1}^{k} S_{j}$.

Our strategy is using Bezier curves to approximate the solutions $y_{j}(t)$ by $v_{j}(t)$ where $v_{j}(t)$ is given below. Individual Bezier curves that are defined over the subintervals are joined together to form the Bezier spline curves. For $j=$ $1,2, \ldots, k$, define the Bezier polynomials $v_{j}(t)$ of degree $n$ that approximate the action of $y_{j}(t)$ over the interval $\left[t_{j-1}, t_{j}\right]$ as follows:

$$
v_{j}(t)=\sum_{r=0}^{n} a_{r}^{j} B_{r, n}\left(\frac{t-t_{j-1}}{h}\right),
$$

where

$$
B_{r, n}\left(\frac{t-t_{j-1}}{h}\right)=\left(\begin{array}{l}
n \\
r
\end{array}\right) \frac{1}{h^{n}}\left(t_{j}-t\right)^{n-r}\left(t-t_{j-1}\right)^{r}
$$

is the Bernstein polynomial of degree $n$ over the interval $\left[t_{j-1}, t_{j}\right]$ and $a_{r}^{j}$ is the control points (see [17]). By substituting (5) in (3), one may define $R_{1, j}(t)$ for $t \in\left[t_{j-1}, t_{j}\right]$ as

$$
R_{1, j}(t)=\epsilon v_{j}^{\prime \prime}(t)+a(t) v_{j}^{-k_{2}+j}(t-\delta)+b(t) v_{j}(t)-f(t) .
$$

Let $v(t)=\sum_{j=1}^{k} \chi_{j}^{1}(t) v_{j}(t)$ where $\chi_{j}^{1}(t)$ is the characteristic function of $v_{j}(t)$ for $t \in\left[t_{j-1}, t_{j}\right]$. Beside the boundary conditions on $v(t)$, at each node, we need to impose continuity condition on each successive pair of $v_{j}(t)$ to guarantee the smoothness. Since the differential equation is of first order, the continuity of $y$ (or $v$ ) and its first derivative give

$$
v_{j}^{(s)}\left(t_{j}\right)=v_{j+1}^{(s)}\left(t_{j}\right), \quad s=0,1, j=1,2, \ldots, k-1,
$$

where $v_{j}^{(s)}\left(t_{j}\right)$ is the $s$ th derivative $v_{j}(t)$ with respect to $t$ at $t=t_{j}$.

Thus, the vector of control points $a_{r}^{j}(r=0,1, n-1, n)$ must satisfy (see [17])

$$
\begin{gathered}
a_{n}^{j}\left(t_{j}-t_{j-1}\right)^{n}=a_{0}^{j+1}\left(t_{j+1}-t_{j}\right)^{n}, \\
\left(a_{n}^{j}-a_{n-1}^{j}\right)\left(t_{j}-t_{j-1}\right)^{n-1}=\left(a_{1}^{j+1}-a_{0}^{j+1}\right)\left(t_{j+1}-t_{j}\right)^{n-1} .
\end{gathered}
$$

Ghomanjani et al. [17] proved the convergence of this method where $h \rightarrow 0$.

Now, the residual function can be defined in $S_{j}$ as follow:

$$
R_{j}=\int_{t_{j-1}}^{t_{j}}\left\|R_{1, j}(t)\right\|^{2} d t,
$$

where $\|\cdot\|$ is the Euclidean norm and $M$ is a sufficiently large penalty parameter. Our aim is solving the following problem over $S=\bigcup_{j=1}^{k} S_{j}$ :

$$
\begin{array}{ll}
\min & \sum_{j=1}^{k} R_{j} \\
\text { s.t. } & a_{n}^{j}\left(t_{j}-t_{j-1}\right)^{n}=a_{0}^{j+1}\left(t_{j+1}-t_{j}\right)^{n}, \\
& \left(a_{n}^{j}-a_{n-1}^{j}\right)\left(t_{j}-t_{j-1}\right)^{n-1} \\
& =\left(a_{1}^{j+1}-a_{0}^{j+1}\right)\left(t_{j+1}-t_{j}\right)^{n-1}, \\
& v_{j}(t)=\phi(t), \quad-\delta \leq t \leq t_{0}, \quad j=1,2, \ldots, k, \\
& v_{k}\left(t_{f}\right)=\gamma .
\end{array}
$$

The mathematical programming problem (11) can be solved by many subroutine algorithms. Here, we use Maple 12 to solve this optimization problem.

\section{Numerical Results and Discussion}

Consider the following examples which can be solved by using the presented method.

Example 1. First we consider the problem (see [11])

$$
\epsilon y^{\prime \prime}(t)+y^{\prime}(t-\delta)-y(t)=0, \quad 0<t<1,
$$

under the boundary conditions

$$
\begin{gathered}
y(t)=1, \quad-\delta \leq t \leq 0, \\
y(1)=1 .
\end{gathered}
$$


TABLE 1: The maximum error for $\epsilon=0.1$ and for different $\delta$ for Example 1.

\begin{tabular}{lcc}
\hline$\delta$ & Max error in [11] & Max error of presented method \\
\hline 0.01 & 0.01182463 & 0.0045 \\
0.03 & 0.01515596 & 0.0090 \\
0.06 & 0.02584799 & 0.0070 \\
0.08 & 0.08313177 & 0.0300 \\
\hline
\end{tabular}

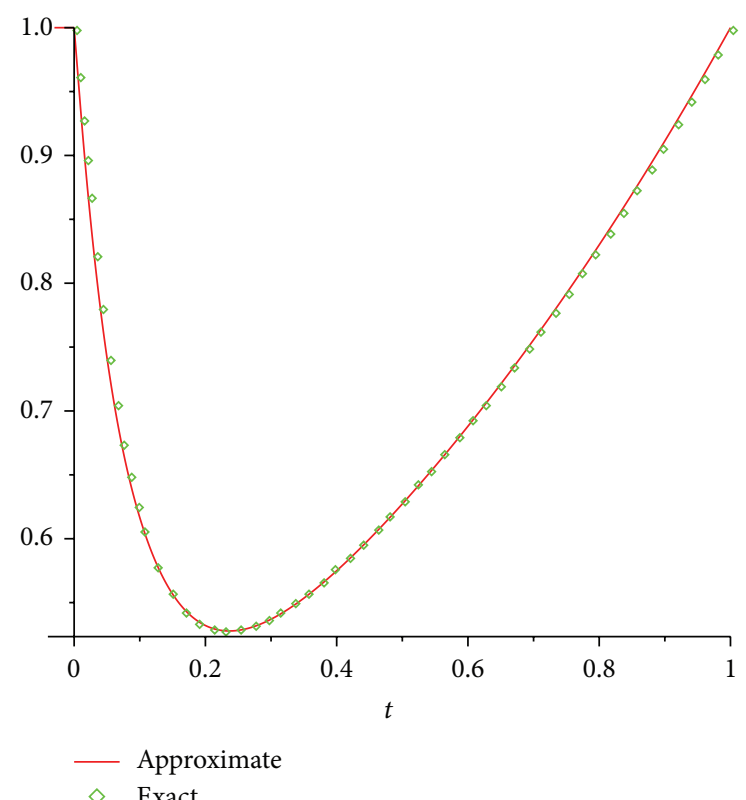

FIGURE 1: Graphs of the exact and computed solution of the BVP with $\epsilon=0.1$ and $\delta=0.01$ for Example 1 .

A boundary layer exists on left side of the interval. For this problem, the exact solution is

$$
y(t)=\frac{\left(1-e^{m_{2}}\right) e^{m_{1} t}+\left(e^{m_{1}}-1\right) e^{m_{2} t}}{\left(e^{m_{1}}-e^{m_{2}}\right)}
$$

where

$$
\begin{aligned}
& m_{1}=\frac{-1-\sqrt{1+4(\epsilon-\delta)}}{2(\epsilon-\delta)}, \\
& m_{2}=\frac{-1+\sqrt{1+4(\epsilon-\delta)}}{2(\epsilon-\delta)} .
\end{aligned}
$$

Also, we have plotted the graphs of the exact and computed solution of the problem in Figure 1. The maximum errors are shown in Table 1.

Example 2. Next we consider the problem (see [11])

$$
\epsilon y^{\prime \prime}(t)-y^{\prime}(t-\delta)-y(t)=0, \quad 0<x<1,
$$

under the boundary conditions

$$
\begin{gathered}
y(t)=1, \quad-\delta \leq t \leq 0, \\
y(1)=-1 .
\end{gathered}
$$

TABLE 2: The maximum error for $\epsilon=0.1$ and for different $\delta$ for Example 2.

\begin{tabular}{lc}
\hline$\delta$ & Max error of presented method \\
\hline 0.01 & 0.007 \\
0.03 & 0.022 \\
0.06 & 0.023 \\
0.08 & 0.025 \\
\hline
\end{tabular}

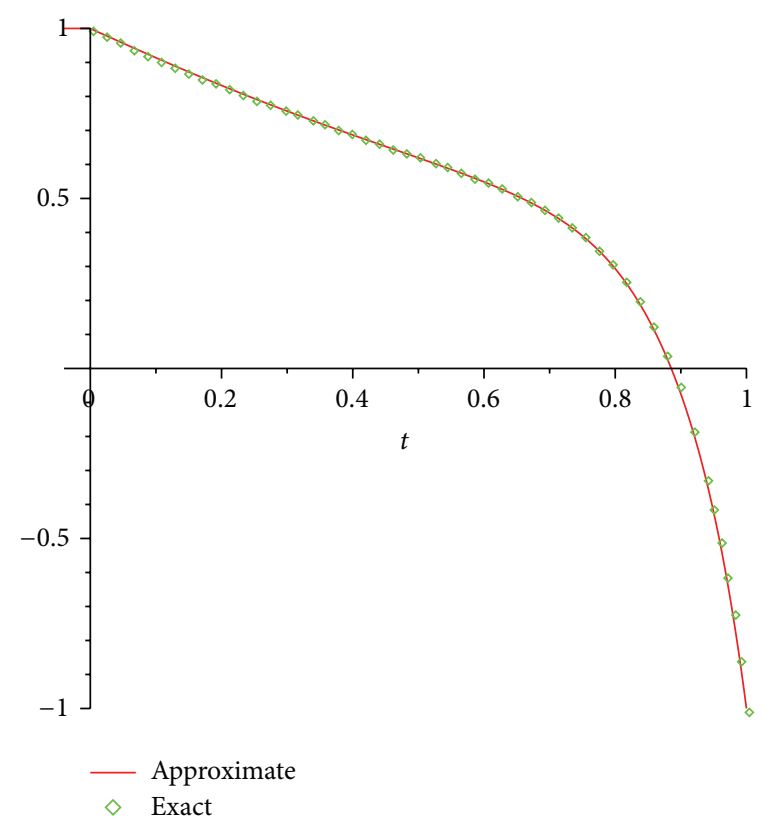

FIGURE 2: Graphs of the exact and computed solution of the BVP with $\epsilon=0.1$ and $\delta=0.01$ for Example 2 .

A boundary layer exists on right side of the interval. For this problem, the exact solution is

$$
y(t)=\frac{\left(1+e^{m_{2}}\right) e^{m_{1} t}-\left(1+e^{m_{1}}\right) e^{m_{2} t}}{\left(e^{m_{2}}-e^{m_{1}}\right)},
$$

where

$$
\begin{aligned}
& m_{1}=\frac{1-\sqrt{1+4(\epsilon+\delta)}}{2(\epsilon+\delta)}, \\
& m_{2}=\frac{1+\sqrt{1+4(\epsilon+\delta)}}{2(\epsilon+\delta)}
\end{aligned}
$$

Also, we have plotted the graphs of the exact and computed solution of the problem in Figure 2. The maximum errors are shown in Table 2.

\section{Conclusions}

We have described a numerical algorithm for solving BVPs for singularly perturbed differential-difference equation with small shifts. Here, we have discussed both the cases by using Bezier curves, when boundary layer is on the left side and when boundary layer is on the right side of the underlying 
interval. Numerical examples show that the proposed method is efficient and very easy to use.

\section{Conflict of Interests}

The authors declare that they have no conflict of interests regarding the publication of this paper.

\section{Acknowledgment}

The authors would like to thank the anonymous reviewers for their careful reading, constructive comments, and nice suggestions which have improved the paper very much.

\section{References}

[1] K. C. Patidar and K. K. Sharma, " $\epsilon$-uniformly convergent nonstandard finite difference methods for singularly perturbed differential difference equations with small delay," Applied Mathematics and Computation, vol. 175, no. 1, pp. 864-890, 2006.

[2] M. K. Kadalbajoo and K. K. Sharma, "Numerical treatment for singularly perturbed nonlinear differential difference equations with negative shift," Nonlinear Analysis: Theory, Methods and Applications, vol. 63, no. 5, pp. e1909-e1924, 2005.

[3] M. K. Kadalbajoo and K. K. Sharma, "Parameter-uniform fitted mesh method for singularly perturbed delay differential equations with layer behavior," Electronic Transactions on Numerical Analysis, vol. 23, pp. 180-201, 2006.

[4] P. Rai and K. K. Sharma, "Numerical analysis of singularly perturbed delay differential turning point problem," Applied Mathematics and Computation, vol. 218, no. 7, pp. 3483-3498, 2011.

[5] C. G. Lange and R. M. Miura, "Singular perturbation analysis of boundary value problems for differential-difference equations. V: small shifts with layer behavior," SIAM Journal on Applied Mathematics, vol. 54, no. 1, pp. 249-272, 1994.

[6] M. C. Mackey and L. Glass, "Oscillations and chaos in physiological control system," Science, vol. 197, pp. 287-289, 1997.

[7] A. Longtin and J. G. Milton, "Complex oscillations in the human pupil light reflex with "mixed" and delayed feedback," Mathematical Biosciences, vol. 90, no. 1-2, pp. 183-199, 1988.

[8] V. Y. Glizer, "Asymptotic analysis and solution of a finitehorizon $H_{\infty}$ control problem for singularly-perturbed linear systems with small state delay," Journal of Optimization Theory and Applications, vol. 117, no. 2, pp. 295-325, 2003.

[9] C. G. Lange and R. M. Miura, "Singular perturbation analysis of boundary value problems for differential-difference equations," SIAM Journal on Applied Mathematics, vol. 42, no. 3, pp. 502531, 1982.

[10] C. G. Lange and R. M. Miura, "Singular perturbation analysis of boundary value problems for differential-difference equations. VI: small shifts with rapid oscillations," SIAM Journal on Applied Mathematics, vol. 54, no. 1, pp. 273-283, 1994.

[11] M. K. Kadalbajoo and K. K. Sharma, "Numerical analysis of singularly perturbed delay differential equations with layer behavior," Applied Mathematics and Computation, vol. 157, no. 1, pp. 11-28, 2004.

[12] M. K. Kadalbajoo and K. K. Sharma, "Numerical treatment of a mathematical model arising from a model of neuronal variability," Journal of Mathematical Analysis and Applications, vol. 307, no. 2, pp. 606-627, 2005.

[13] M. K. Kadalbajoo and K. K. Sharma, "A numerical method based on finite difference for boundary value problems for singularly perturbed delay differential equations," Applied Mathematics and Computation, vol. 197, no. 2, pp. 692-707, 2008.

[14] P. Rai and K. K. Sharma, "Numerical study of singularly perturbed differential-difference equation arising in the modeling of neuronal variability," Computers \& Mathematics with Applications, vol. 63, no. 1, pp. 118-132, 2012.

[15] G. M. Amiraliyev and F. Erdogan, "Uniform numerical method for singularly perturbed delay differential equations," Computers \& Mathematics with Applications, vol. 53, no. 8, pp. 1251-1259, 2007.

[16] I. G. Amiraliyeva and G. M. Amiraliyev, "Uniform difference method for parameterized singularly perturbed delay differential equations," Numerical Algorithms, vol. 52, no. 4, pp. 509-521, 2009.

[17] F. Ghomanjani, M. H. Farahi, and M. Gachpazan, "Bézier control points method to solve constrained quadratic optimal control of time varying linear systems," Computational \& Applied Mathematics, vol. 31, no. 3, pp. 433-456, 2012.

[18] F. Ghomanjani, A. Kılıçman, and S. Effati, "Numerical solution for IVP in Volterra type linear integro-differential equations system," Abstract and Applied Analysis, vol. 2013, Article ID 490689, 4 pages, 2013. 


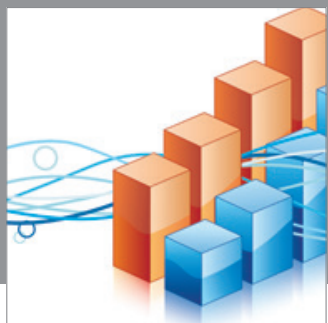

Advances in

Operations Research

mansans

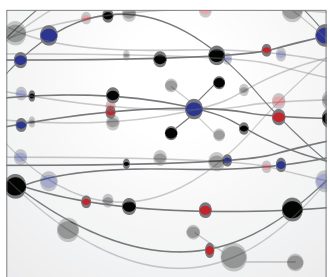

The Scientific World Journal
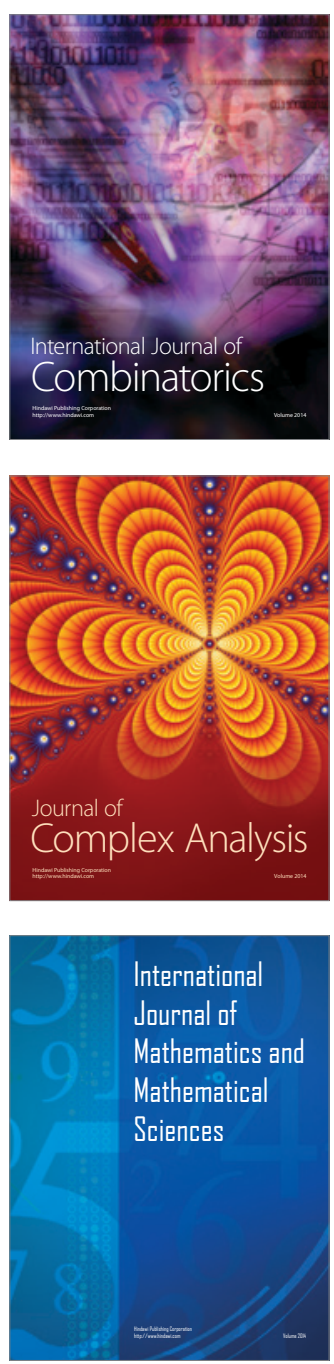
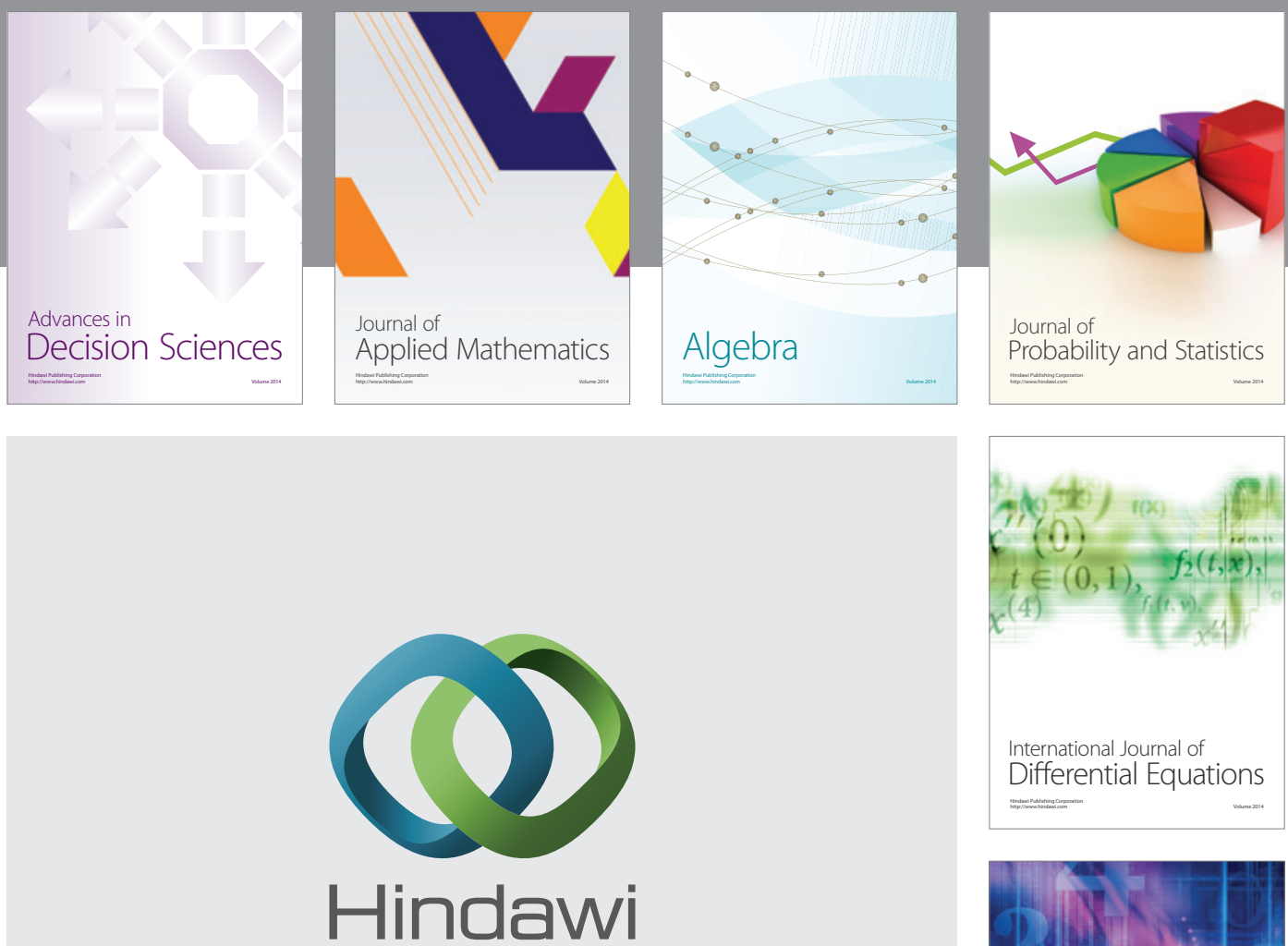

Submit your manuscripts at http://www.hindawi.com
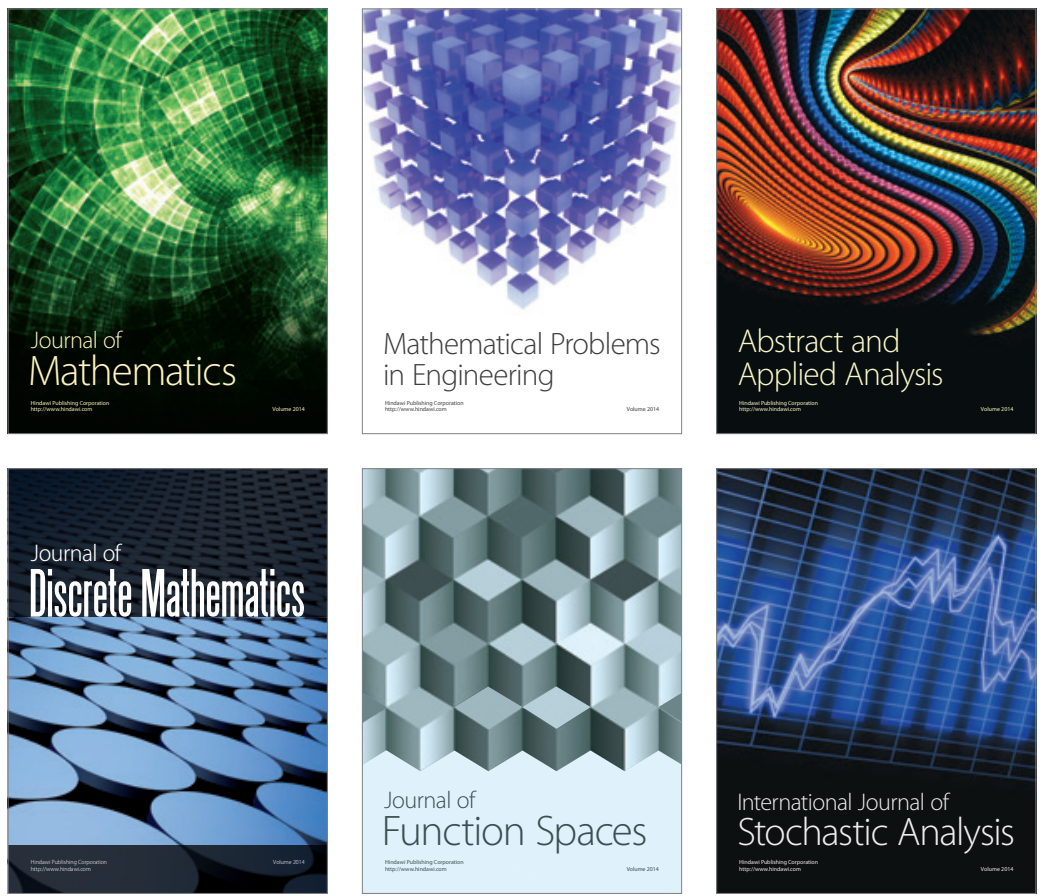

Journal of

Function Spaces

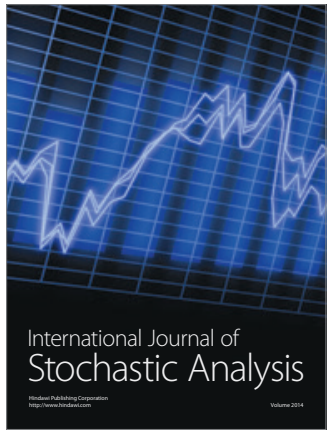

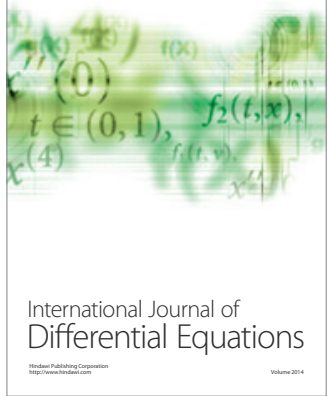
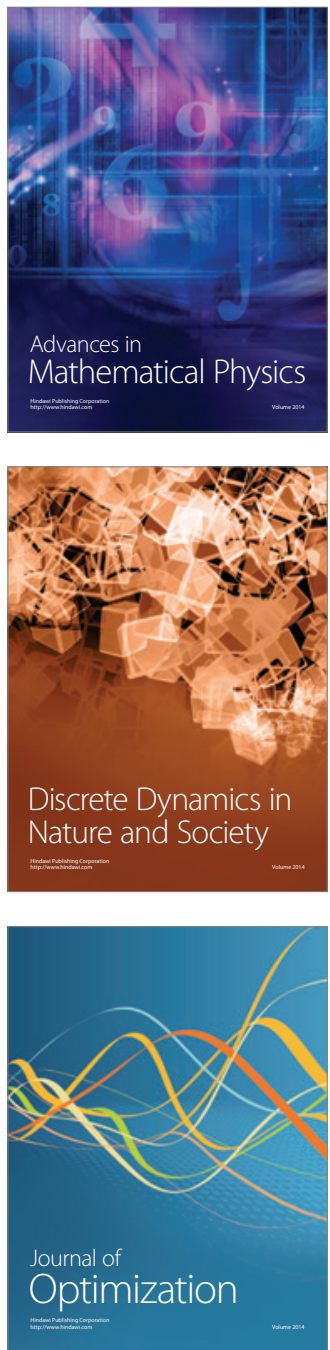